\title{
Constraints on the Low-Energy Cutoff in the Electron Distribution of the PKS 0637-752 Jet
}

\author{
M. Mueller \\ Stanford Linear Accelerator Center, 2575 Sand Hill Road, Menlo Park, CA 94025, \\ and Kavli Institute for Particle Astrophysics and Cosmology, Stanford University, \\ Stanford, CA 94305 \\ mmueller@slac.stanford.edu \\ and \\ D. A. Schwartz \\ Harvard-Smithsonian Center for Astrophysics, 60 Garden Street, Cambridge, MA 02138 \\ das@head.cfa.harvard.edu
}

\begin{abstract}
We re-analyze the Chandra ACIS spectrum of the kpc-scale jet in PKS 0637-752 to investigate the possible low energy cutoff in the relativistic electron spectrum producing the non-thermal radiation in the scenario of inverse Compton emission off the cosmic microwave background. This was among the first objects targeted by the Chandra Observatory and gives a unique opportunity to study the low energy X-ray emission free of detector contamination. As previously reported in the literature, the spectrum can be fit by a power law, with the slope predicted by the radio spectrum, modified by low energy absorption through the Galaxy as determined from the spectrum of the quasar core and by HI $21 \mathrm{~cm}$ observations. We obtain a marginally better fit with an model of inverse Compton emission produced by an electron population that exhibits a cutoff at $\gamma_{\min } \delta_{10}$ between about 50 and 80 (assuming $\Gamma=\delta$ ). This range for $\gamma_{\text {min }}$ is higher than has previously been assumed in broad-band spectral fits to the jet emission. The observed optical flux can be used to place a lower limit on $\gamma_{\text {min }}$; the constraint is not very strong, but does suggest that $\gamma_{\text {min }}$ must be higher than 1 to avoid overproducing the optical emission. We investigate the effect of uncertainties in the column density for galactic absorption as well as the calibration of Chandra for these early observations. Finally, we discuss the implication of these limits on the jet luminosity in this source.
\end{abstract}


Subject headings: galaxies: jets - quasars: individual (PKS 0637-752)-X-rays: galaxies

\section{INTRODUCTION}

The initial observations to focus Chandra using the presumed point source PKS 0637-752, a quasar at redshift 0.651 (Savage. Browne. \& Bolton 1976), revealed a remarkable X-ray jet structure (Schwartz et al. 2000; Chartas et al. 2000), coincident with the radio jet reported by Tingay et al. (1998). Schwartz et al. (2000) pointed out that the X-ray emission could not be explained reasonably by thermal bremsstrahlung, by an extension of the radio synchrotron spectrum, by synchrotron self-Compton, or by inverse-Compton (IC) from any likely source of seed photons provided that the magnetic field was near its equipartition value. Nonetheless they concluded that IC from the same electrons producing the radio emission was the most likely source of the X-rays in view of the similarity of the X-ray and radio surface brightness profile.

Tavecchio et al. (2000) and Celotti. Ghisellini \& Chiaberge (2001) solved the dilemma by invoking bulk relativistic motion of the $100 \mathrm{kpc}$ scale jet. Relativistic motion with bulk Lorentz factor $\Gamma$ has the effect of requiring a smaller magnetic field in the rest frame for minimum energy conditions, and of increasing the apparent energy density of the cosmic microwave background (CMB) by a factor of $\Gamma^{2}$ on average as seen by the radiating electrons. Such an IC/CMB explanation subsequently has been applied successfully to explain

the X-ray emission from powerful quasar and FR II radio jets; e.g., by Sambruna et al. (2002); Harris \& Krawczvnski (2002); Siemiginowska et al. (2002, 2003); Sambruna et al. (2004); Marshall et al. (2005); Schwartz et al. (2006); Sambruna et al. (2006) and Schwartz (2007).

Tavecchio et al. (2000) pointed out that the scenario in which low energy electrons underwent Compton scattering on an external photon field gave an opportunity to infer parameters of the low energy portion of the relativistic electron distribution for the first time. They estimated $\gamma_{\min } \sim 10$ for the PKS $0637-752$ jet, by constructing a fiducial spectral model which fit the broad-band radio, optical and X-ray flux data. Subsequent examinations of the jet spectrum (Celotti, Ghisellini \& Chiaberge 2001; Uchiyama et al. 2005) all converged on a value for $\gamma_{\min } \sim 10-20$. In the present work we perform a more rigorous assessment of $\gamma_{\min }$ by performing a detailed spectral fitting of the ACIS X-ray spectrum. PKS 0637-752 is in the unique position among identified X-ray jet sources in that, because the exposures were done so early in the lifetime of Chandra, the low-energy X-ray spectrum is free of the detector contamination and associated loss of sensitivity that affect later observations. The 
main source of uncertainty remains the low-energy calibration of ACIS, and we contrast results obtained from a wider bandpass $(0.3-7 \mathrm{keV})$ than is usually used for imaging spectral analysis using Chandra with the equivalent results if the bandpass is restricted to the bettercalibrated range of $0.55-7 \mathrm{keV}$.

Section 2 lists the observations and data reduction for this investigation. The development of the custom IC/CMB spectral models is detailed in Section 3, which are then applied to the data in Section 4 to derive limits on $\gamma_{\min }$. The interpretation of the model fits, including a comparison between two different prescriptions for the calculation of the IC/CMB spectrum and a discussion of the effect of the shape of the electron distribution at the $\gamma_{\min }$ cut-off, is the topic of Section 5. Section 6 summarizes the paper.

\section{OBSERVATIONS AND DATA REDUCTION}

PKS 0637-752 was observed by Chandra using the ACIS-S instrument as part of the initial focusing period. This early in the mission, there was no loss of low-energy sensitivity due to the contamination layer. We thus have an opportunity with this object to push the $\mathrm{X}$-ray spectrum to lower energies than is currently possible.

Table 1 lists the details of the Chandra observations that were used in this investigation, all of which were carried out in the FAINT TIMED mode of ACIS. We selected the observations based on whether the quasar and jet were positioned on the back-illuminated S3 chip, which excludes two observations (ObsID 1059 and 1061).

Other observations were discarded: ObsID 1057 and 62558 because the identification of the quasar and jet was unclear, and ObsID 1093, 1264, and 1265 since they do not appear to contain any useful data. Although most of the remaining observations were not at the best focus, the quasar and jet are clearly separated, and the jet spectrum can be extracted without contributions from the quasar core.

For each observation, the evt2 file was re-extracted from the evt1 file in order to apply the latest calibrations, as outlined in the ACIS data preparation thread on the CIAO web page (CALDB version 3.2.4). Since the observations were taken early in the mission, and the focal temperature was different from $-120^{\circ} \mathrm{C}$, no time-dependent gain or CTI correction was used.

Extraction regions for the jet and background were defined individually for each observation, since in some cases the departure from optimal focus necessitated a larger extraction region to include all events from the jet. The extraction regions were divided into an inner 
and outer jet, the first of which included the faint X-ray emission of the bridge between the quasar core and the bright radio/optical/X-ray knots, which comprised the second region. The regions for one of the observations (ObsID 62554) are shown in Figure 1, No significant differences in the spectra between these two sub-regions were found, although the low number of counts in the bridge spectrum make a detailed comparison difficult. All subsequent fits used only the outer jet extraction region.

Using the CIAO tool dmextract, the counts in each observation's source and background extraction regions were combined into pulse invariant (PI) channels to form the individual spectra. A grouping algorithm was applied to have a minimum of 2 counts per bin. Weighted ancillary response files (WARF) and redistribution matrices (WRMF) were created using the tools mkwarf and mkrmf, as appropriate for extended sources. (mkacisrmf does not apply for these observations due to their early observation dates.) To check for background flares, the light curve of the S3 chip excluding the region around the quasar and the jet was analyzed visually. Data during times corresponding to the few occurrences of spikes in the count rate were filtered out in the extraction.

Because the results reported in this investigation depend heavily on the correct modeling of the low-energy response of acis, alternative calibration products were extracted to investigate the effect of uncertainties in the calibration (Grant, C. \& Bautz, M., private communication). Sets of WARF and WRMF were constructed using $-100^{\circ} \mathrm{C}$ and $-120^{\circ} \mathrm{C}$ FEF files. In addition, for the $-100^{\circ} \mathrm{C} \mathrm{FEF} \mathrm{calibration} \mathrm{products,} \mathrm{the} \mathrm{Quantum} \mathrm{Efficiency}$ Uniformity file was varied between the flat version N0001 and the version N0002 that includes information on how the quantum efficiency varies over the S3 chip. Spectral fits were repeated with both the nominal set of calibration files and the above alternative calibrations. The results on $\gamma_{\text {min }}$ are unaffected to within the uncertainties introduced by the choice of IC scattering kernel and the shape of the $\gamma_{\min }$ cutoff (see Section 3). However, we will report results obtained from both a $0.3-7 \mathrm{keV}$ bandpass as well as a better-calibrated $0.55-7 \mathrm{keV}$ bandpass.

PKS 0637-752 was also observed by the Hubble Space Telescope (Schwartz et al. 2000). The area where the three optical knots are located corresponds morphologically to the extraction region used for the X-ray analysis. The total optical flux density from the knots is $0.574 \mu \mathrm{Jy}$ at a frequency of $4.3 \times 10^{14} \mathrm{~Hz}$. 


\section{IC/CMB MODEL}

We wish to fit the spectrum for the jet with a model of inverse Compton scattering of energetic electrons on the CMB. We assume that the spectrum of the entire X-ray jet is emitted by a spatially homogeneous population of electrons that collectively exhibit a bulk motion with Lorentz factor $\Gamma=\left(1-\beta^{2}\right)^{-1 / 2}$. The random motion of the electrons (assumed isotropic in the jet rest frame) is described by the distribution of the Lorentz factor $\gamma$, such that the energy of any given electron in the jet rest frame is $\gamma m_{e} c^{2}\left(m_{e}\right.$ : electron rest mass, $c$ : speed of light; primed quantities refer to the jet rest frame, unprimed to the observer frame; exception: $\gamma$, which always refers to the jet rest frame).

This distribution is modeled as a power law between $\gamma_{\min }$ and $\gamma_{\max }$, with a slope $s$ $\left(n^{\prime}(\gamma) \propto \gamma^{-s}\right)$. The upper end of the distribution is chosen as a sharp cutoff; since we note that the high energy cutoff is not reached in the X-ray spectrum, the exact functional form doesn't affect the analysis as long as $\gamma_{\max }$ is chosen sufficiently high. The shape of the low-energy cutoff, however, has a direct effect on the model spectrum. We investigate two options: a step function at $\gamma_{\min }$ :

$$
n^{\prime}(\gamma)= \begin{cases}N_{0}^{\prime} \gamma^{-s} & \gamma_{\min } \leq \gamma<\gamma_{\max } \\ 0 & \text { otherwise }\end{cases}
$$

and a constant electron density below $\gamma_{\min }$ :

$$
n^{\prime}(\gamma)= \begin{cases}N_{0}^{\prime} \gamma_{\min }^{-s} & \gamma<\gamma_{\min } \\ N_{0}^{\prime} \gamma^{-s} & \gamma_{\min } \leq \gamma<\gamma_{\max } \\ 0 & \gamma_{\max } \leq \gamma .\end{cases}
$$

Neither of them is likely to be the actual shape of the low-energy cutoff, but they (together with the case when $\gamma_{\min }$ is sufficiently small as to move the cutoff outside of the spectrum bandpass) provide three representative cases for the characterization of the electron distribution based on the available X-ray data.

$j^{\prime}\left(E_{1}^{\prime}, \Omega_{1}^{\prime}\right)$, the IC/CMB emissivity in the jet rest frame at a given energy $E_{1}^{\prime}$ and direction $\Omega_{1}^{\prime}$, can be formulated as an integral over the invident electron and photon energies and their directions, taking into account their respective number densities (which are themselves functions of the corresponding particle's energy) and the relativistic transformations of the energies and directions between the jet rest frame and the individual electron's rest frames. The scattering of CMB photons into the X-ray band is due to electrons with Lorentz factors small enough to make the scattering event in the electron rest frame be safely within the 
Thomson limit. This and other assumptions allow these integrals to be evaluated in terms of elementary functions. We wish to compare two different approaches that differ in the way the photon field is treated.

\subsection{Blumenthal \& Gould Approach}

The Blumenthal \& Gould (1970) calculation assumes an isotropic distribution of seed photon directions, which is strictly speaking not applicable to a jet moving relativistically with respect to the $\mathrm{CMB}$ rest frame. However, the simplicity of the resulting expression for the emissivity is the reason it has been used extensively in the literature. Since both the electron and photon fields are treated as isotropic, the emissivity does not depend on $\Omega_{1}^{\prime}$ and can be written as

$$
j^{\prime}\left(E_{1}^{\prime}\right) \propto E_{1}^{\prime} \int_{\gamma_{\text {crit }}}^{\gamma_{\max }} \int_{E_{0}^{\prime}} \frac{n^{\prime}(\gamma)}{\gamma^{2}} f\left(E_{0}^{\prime}, E_{1}^{\prime}, \gamma\right) \mathrm{d} E_{0}^{\prime} \mathrm{d} \gamma
$$

where $f\left(E_{0}^{\prime}, E_{1}^{\prime}, \gamma\right)=2 x \ln x-2 x^{2}+x+1$, with $x=E_{1}^{\prime} /\left(4 \gamma^{2} E_{0}^{\prime}\right) . \gamma \gg 1$ is assumed throughout. The function $f$ is often called the kernel of IC scattering, because it describes the spectrum obtained from a fixed initial electron and photon energy. $\gamma_{\text {crit }}$, which is the Lorentz factor of the least-energetic electrons that can contribute to the emission at $E_{1}^{\prime}$, is derived as

$$
\gamma_{\text {crit }}=\frac{1}{2} \sqrt{\frac{E_{1}^{\prime}}{E_{0}^{\prime}}}
$$

A further simplification treats the incident CMB photon field as monoenergetic at an energy $E_{0}^{\prime}=(1+z) \Gamma \sigma k T_{\mathrm{CMB}}$, where $\sigma=2.7$ is the average photon energy of a thermal spectrum in units of $k T$ (Felten \& Morrison 1966), $k$ is the Boltzmann constant, and $T_{\mathrm{CMB}}=$ $2.725 \mathrm{~K}$ is the local CMB temperature (Fixsen et al. 1996). The integral over $E_{0}^{\prime}$ in Equation 3 is thus eliminated. The motivation for this simplification is that features in the observed $\mathrm{X}$-ray spectrum are expected to be much broader than the width of the thermal distribution of CMB photon energies.

In the case of the electron distribution with the sharp cut off at $\gamma_{\min }$ (Equation 1), the final expression for the emissivity is 


$$
j^{\prime}\left(E_{1}^{\prime}\right) \propto E_{1}^{\prime} \int_{\max \left(\gamma_{\text {crit }}, \gamma_{\min }\right)}^{\gamma_{\max }} \gamma^{-s-2} f(x) \mathrm{d} \gamma .
$$

For the other electron distribution (Equation 2), the equivalent expression is

$$
j^{\prime}\left(E_{1}^{\prime}\right) \propto E_{1}^{\prime} \int_{\gamma_{\text {crit }}}^{\gamma_{\max }} \gamma^{-s-2} f(x) \mathrm{d} \gamma
$$

if $\gamma_{\text {crit }} \geq \gamma_{\min }$, and

$$
j^{\prime}\left(E_{1}^{\prime}\right) \propto E_{1}^{\prime} \int_{\gamma_{\min }}^{\gamma_{\max }} \gamma^{-s-2} f(x) \mathrm{d} \gamma+E_{1}^{\prime} \gamma_{\min }^{-s} \int_{\gamma_{\text {crit }}}^{\gamma_{\min }} \gamma^{-2} f(x) \mathrm{d} \gamma,
$$

if $\gamma_{\text {crit }}<\gamma_{\min }$.

The observed spectral flux $j\left(E_{1}\right)$ is proportional to the emissivity in the jet rest frame $j^{\prime}\left(E_{1}^{\prime}\right)$ when the energy shift of the photons due to the motion of the jet rest frame with respect to the observer is taken into account. Photons emitted at an energy $E_{1}^{\prime}$ in the jet rest frame are observed at an energy $E_{1}=\delta E_{1}^{\prime} /(1+z)$ in the observer frame, where $\delta=[\Gamma(1-\beta \mu)]^{-1}$ is the jet Doppler factor (with $\theta=\cos ^{-1} \mu$ the observer viewing angle of the jet) and $z$ is the redshift of the quasar. Since for the determination of $\gamma_{\min }$ we are only interested in the shape of the spectrum, and not its normalization, the implementation of the model simply uses the jet rest frame emissivity appropriately shifted along the energy axis and applies a normalization factor that is not specified in detail to best fit the observed number of counts.

\subsection{Aharonian \& Atoyan Approach}

In contrast to Blumenthal \& Gould (1970), the Aharonian \& Atovan (1981) approach treats the incident photon field as monodirectional (anti-parallel to the jet bulk velocity for IC/CMB) in the jet rest frame, which is an appropriate approximation for $\Gamma \gg 1$. Because the electron distribution in the jet rest frame is assumed isotropic, the system now exhibits azimuthal symmetry around the propagation direction of the jet, and the angular dependence of the emissivity reduces to a dependence on the polar angle $\theta^{\prime}=\cos ^{-1} \mu^{\prime}$ of the outgoing photon. If $\gamma \gg 1$ is assumed as well, then, irrespective of the scattering angle in the electron rest frame, the direction (in the jet rest frame) of the photon after scattering is well approximated by the direction of the incident electron. The integration over the incident electron direction thus reduces to a $\delta$-function substitution. 
Adopting the notation of Stawarz et al. (2005), the emissivity can be written as

$$
j^{\prime}\left(E_{1}^{\prime}, \mu^{\prime}\right) \propto E_{1}^{\prime} \int \gamma_{\text {crit }} \gamma_{\max } \mathrm{d} \gamma \int \mathrm{d} E_{0}^{\prime} \frac{n^{\prime}(\gamma)}{\gamma^{2}} f\left(E_{0}^{\prime}, E_{1}^{\prime}, \gamma, \mu^{\prime}\right)
$$

with the IC kernel, expressed using the quantities $v^{\prime}=2\left(1-\mu^{\prime}\right) E_{0}^{\prime} \gamma /\left(m_{e} c^{2}\right)$ and $w^{\prime}=$ $E_{1}^{\prime} /\left(\gamma m_{e} c^{2}\right)$

$$
f\left(E_{0}^{\prime}, E_{1}^{\prime}, \gamma, \mu^{\prime}\right)=1+\frac{w^{\prime 2}}{2\left(1-w^{\prime}\right)}-\frac{2 w^{\prime}}{v^{\prime}\left(1-w^{\prime}\right)}+\frac{2 w^{\prime 2}}{v^{\prime 2}\left(1-w^{\prime}\right)^{2}}
$$

In this case, $\gamma_{\text {crit }}$ evaluates to

$$
\gamma_{\mathrm{crit}}=\frac{E_{1}^{\prime}}{2 m_{e} c^{2}}\left\{1+\left(1+\frac{2 m_{e}^{2} c^{4}}{\left(1-\mu^{\prime}\right) E_{0}^{\prime} E_{1}^{\prime}}\right)^{1 / 2}\right\}
$$

Again, the condition that $\gamma \gg 1$ has to be satisfied. The additional condition in Aharonian \& Atoyan (1981), that $E_{1}^{\prime} \gg E_{0}^{\prime}$, is the same simplifying assumption mentioned in the explanatory text to Equation 7.26a in Rybicki \& Lightman (1979) and to Equation 2.13 in Blumenthal \& Gould (1970) and is readily satisfied for IC/CMB X-rays. While the above expression is derived in the Klein-Nishina regime, the authors stress that the exact formula for the IC spectrum (without the simplifying assumptions mentioned above) is valid for any values of the electron and photon energies. Since the subsequent simplifications are satisfied in the Thomson regime also, Equation 8 is applicable to the process of X-ray generation via IC/CMB. In addition, $f\left(E_{0}^{\prime}, E_{1}^{\prime}, \gamma, \mu^{\prime}\right)$ does not appear to require any modifications to mitigate potential numerical precision issues in its evaluation in the Thomson limit.

In the case of the electron distribution with the sharp cut off at $\gamma_{\min }$ (Equation 1), the final expression for the emissivity is

$$
j^{\prime}\left(E_{1}^{\prime}, \mu^{\prime}\right) \propto E_{1}^{\prime} \int_{\max \left(\gamma_{\text {crit }}, \gamma_{\min }\right)}^{\gamma_{\max }} \gamma^{-s-2} f\left(E_{0}^{\prime}, E_{1}^{\prime}, \gamma, \mu^{\prime}\right) \mathrm{d} \gamma
$$

For the other electron distribution (Equation 2), the equivalent expression is

$$
j^{\prime}\left(E_{1}^{\prime}, \mu^{\prime}\right) \propto E_{1}^{\prime} \int_{\gamma_{\text {crit }}}^{\gamma_{\max }} \gamma^{-s-2} f\left(E_{0}^{\prime}, E_{1}^{\prime}, \gamma, \mu^{\prime}\right) \mathrm{d} \gamma
$$

if $\gamma_{\text {crit }} \geq \gamma_{\min }$, and 


$$
\begin{aligned}
& j^{\prime}\left(E_{1}^{\prime}, \mu^{\prime}\right) \propto E_{1}^{\prime} \int_{\gamma_{\min }}^{\gamma_{\max }} \gamma^{-s-2} f\left(E_{0}^{\prime}, E_{1}^{\prime}, \gamma, \mu^{\prime}\right) \mathrm{d} \gamma+E_{1}^{\prime} \gamma_{\min }^{-s} \int_{\gamma_{\text {crit }}}^{\gamma_{\min }} \gamma^{-2} f\left(E_{0}^{\prime}, E_{1}^{\prime}, \gamma, \mu^{\prime}\right) \mathrm{d} \gamma \\
& \quad \text { if } \gamma_{\text {crit }}<\gamma_{\min } .
\end{aligned}
$$

As in the Blumenthal \& Gould (1970) approach, the observed spectrum $j\left(E_{1}, \mu\right) \propto$ $j^{\prime}\left(E_{1}^{\prime}, \mu^{\prime}\right) . \quad \mu^{\prime}$ is related to $\mu$ and $\beta$ through $\mu^{\prime}=(\beta-\mu) /(1-\beta \mu)$, when the incident photons in the jet rest frame are traveling in the opposite direction to the jet. Since the jet viewing angle is small (Lovell et al. 2000), we assume that $\Gamma=\delta$, which implies $\mu=\beta$, and thus $\mu^{\prime}=0$.

\subsection{Model Implementation}

We proceed to implement custom XsPEC (Arnaud 1996) models that compute spectra based on the above formulas. The numerical integration is done using the qsimp routine in Press et al. (1992). For both approaches, the two adjustable model parameters are $s$ (the electron distribution power law index) and $\gamma_{\min }$. The additional parameters that are not adjusted in the fit are $\gamma_{\max }, \Gamma, \delta$, and $z \cdot \gamma_{\max }$ is kept fixed at $10^{5}$ (corresponding to a high-energy cut-off around $850 \mathrm{MeV}) ; z=0.651$. As mentioned before, the normalization of the model is arbitrary, as the correspondence between the normalization and the relevant physical quantities like luminosity and distance is not spelled out.

Following Tavecchio et al. (2000) and Schwartz (2007), we assume $\Gamma=\delta=10$, which sets $E_{0}^{\prime}=1.05 \times 10^{-5} \mathrm{keV}$. From the $\delta$-function approximation to the energy spectrum of IC scattering from a fixed initial electron and photon energy (where $E_{1}^{\prime}=4 / 3 \gamma^{2} E_{0}^{\prime}$ fixed), the Lorentz factor for electrons scattering CMB seed photons to a given observed energy scales as $(\Gamma \delta)^{-1 / 2}$. Under the assumption that $\Gamma=\delta$, this reduces to a scaling by $\delta^{-1}$. The same behavior is expected for the two kernels used in the models, although this was not investigated in detail. Our quoted results for $\gamma_{\min }$ are thus expected to depend on the assumed value for $\delta$ in the same manner, and we report all results for $\gamma_{\text {min }}$ with this scaling in mind. For reasonable departures from the assumed values for $\Gamma$ and $\delta$, an approximate scaling by $(\Gamma \delta)^{-1 / 2}$ is expected to remain even if $\Gamma=\delta$ is not assumed, since the dominant behavior of the scattering kernel in all cases includes the photon and electron energies in the combination $E_{1}^{\prime} /\left(\gamma^{2} E_{0}^{\prime}\right)$. 


\section{RESULTS}

Given that each individual spectrum has only a small number of counts over the bandpass of interest and was therefore grouped to have a minimum of only two counts per channel,

$\chi^{2}$ is not appropriate as a fitting statistic. Instead, the C-statistic implemented in XSPEC was used. Unfortunately, this statistic does not allow for a goodness-of-fit test, in the way the reduced $\chi^{2}$ is commonly used. However, a visual inspection of the fits reveals a very good agreement between the data and the model. In any case, the important discriminant will be the changes in the fitting statistic with the model parameters as well as between models. All subsequent results were obtained from simultaneous fits to the 21 individual spectra while fixing the model normalization to be the same between the spectra.

\subsection{Phenomenological Fits}

We first fit the spectra in the $0.3-7 \mathrm{keV}$ bandpass with a single power law, modified by neutral Galactic absorption. If $n_{\mathrm{H}}$ is allowed to be free, a best-fit value of $(9.1 \pm 0.8) \times 10^{20}$ $\mathrm{cm}^{-2}$ is returned, and the best-fitting power law energy index is $\alpha=0.76 \pm 0.04$. These values correspond very well with the expected values: the absorbing column with the value reported by Dickey \& Lockman (1990) $\left(9.1 \times 10^{20} \mathrm{~cm}^{-2}\right)$, and the power law index with both the results from earlier investigations of the Chandra spectrum of this source (Chartas et al. 2000, $\alpha=0.85 \pm 0.08$, ) and the radio spectral index (Schwartz et al. 2000). The same power law index (to within uncertainties) is obtained by fixing $n_{\mathrm{H}}=9.1 \times 10^{20} \mathrm{~cm}^{-2}$ before fitting. The best-fit C-statistic value is 886.3 for 958 bins.

In the restricted $0.55-7 \mathrm{keV}$ bandpass, the fit with both the power law index and the absorbing column free returns $n_{\mathrm{H}}=3.0 \times 10^{20} \mathrm{~cm}^{-2}$ (with large uncertainties) and $\alpha=$ $0.63 \pm 0.05$. The C-statistic at the minimum is 808.2 for 860 bins, but it is clear that the low value for the absorption and the resulting fit is spurious, as the restricted bandpass is not very sensitive to the absorption column density. If $n_{\mathrm{H}}=9.1 \times 10^{20} \mathrm{~cm}^{-2}$ is fixed before fitting, the power law index increases to $\alpha=0.84 \pm 0.02$, once more consistent with previous values. The minimum value of the fitting statistic is 817.2 for 860 bins. In all subsequent fits, the absorbing column is kept fixed at the Galactic value.

The normalization of the power law component (before absorption) is in all cases equal to about $(3.1 \pm 0.5) \times 10^{-5}$ photons $/ \mathrm{cm}^{2} / \mathrm{s} / \mathrm{keV}$, corresponding to a flux density of $(21 \pm 3) \mathrm{nJy}$ at $1 \mathrm{keV}$. This is slightly smaller than, but probably within $1 \sigma$ of, previous measurements (Schwartz et al. 2000), which could be due to differences in the extraction region or the background subtraction. 


\subsection{IC/CMB Model Fits}

\subsubsection{Upper Limit on $\gamma_{\min }$ from X-ray Spectrum}

We now wish to investigate whether a better fit can be obtained by letting the IC/CMB spectrum cut off before the low-energy end of the bandpass under consideration. It is expected that if $\gamma_{\min } \delta_{10} \lesssim 40\left(\delta_{10}=\delta / 10\right)$, the cutoff will be too low in energy to affect the 0.3-7 keV bandpass; obviously, this limiting value of $\gamma_{\min }$ will be higher for the $0.55-7 \mathrm{keV}$ bandpass.

We proceed to fit the spectra with the custom IC/CMB models developed in Section 3 ,

For the 0.3-7 keV bandpass, the best fit for both the Blumenthal \& Gould (1970) and the Aharonian \& Atoyan (1981) kernel, and for both the sharp cutoff in the electron distribution or the flat segment case (cases (1) and (2) in Figure 4), is such that the best-fitting $\gamma_{\min }$ is above the limiting value. In fact, plotting the fitting statistic as a function of $\gamma_{\text {min }}$ leads to Figure 2, which shows that a cutoff due to $\gamma_{\min }$ appears to be detected about $2 \sigma$ statistical confidence for two of the four cases. Note that the differences in C-statistic values are equivalent to differences in $\chi^{2}$, such that the same $\Delta \chi^{2}$ values may be used to determine the confidence regions of fitted parameters.

The best-fit values of the fitting statistic and the constraints that we are able to place on $\gamma_{\text {min }}$ are shown in Table 2, In all cases, the fit is as good as the unbroken power law fit or better. The y-axis in Figure 2 is normalized to have a $\Delta$ (C-statistic) of 0 for the best fit. We expect the previous fit to the unbroken power law to be recovered by pushing $\gamma_{\min }$ below the limiting value. In detail, the IC/CMB models to not revert exactly to a power law even if $\gamma_{\min }$ is very low; furthermore, slight differences in the value of the fitting statistic are expected near the minimum, given the minimization algorithm employed by XSPEC. The limiting value of the fitting statistic as $\gamma_{\min } \rightarrow 1$ can therefore be slightly different from the value obtained for the unbroken power law fit above.

The power law index for the electron distribution is returned in all four cases as $s=$ $2.6 \pm 0.1$, consistent with the measurement of the energy index measured for the power law fit $(\alpha=(s-1) / 2)$. The index is systematically lower for the case of the flat electron distribution, but the difference to the index for the sharp cutoff in the electron distribution is not statistically significant. There is almost no degeneracy between the power law index and $\gamma_{\min }$.

As expected, restricting the analysis to the $0.55-7 \mathrm{keV}$ bandpass eliminates the minimum in the fitting statistic, and only upper limits on $\gamma_{\min }$ are obtained. The behavior of the fitting statistic as a function of $\gamma_{\min }$ for the restricted bandpass is shown in Figure 3. Again, the 
relevant data on the best fits and on the $\gamma_{\min }$ constraints are included in Table 2 ,

It is worth noting that, contrary to expectations, the upper limits on $\gamma_{\text {min }}$ from the restricted bandpass are as tight as from the full $0.3-7 \mathrm{keV}$ bandpass. The reason for this might be that the residuals to the fit in the restricted bandpass favor an unbroken power law down to the lowest bins and are very sensitive to changes in the shape at the low-energy end, while the residuals in the bins between $0.3-0.55 \mathrm{keV}$ are more ambivalent about the shape of the model in that range.

\subsubsection{Lower Limit on $\gamma_{\min }$ from Optical Flux Measurement}

Figure 4 shows the optical and X-ray measurements of PKS 0637-752 as well as the predicted IC/CMB spectra based on $\gamma_{\min } \delta_{10}=60$ and the best-fit value of the power law index $s=2.6$. As $\gamma_{\min }$ becomes smaller, the low-energy extension of the model fitted to the X-ray observations will approach and at some point over-predict the observed optical flux (Schwartz et al. 2000). This gives us an opportunity to place lower limits on $\gamma_{\text {min }}$.

The sum of the individual knots' optical flux density is $0.574 \mu \mathrm{Jy}$, and we estimate a conservative error on that measurement of $0.1 \mu \mathrm{Jy}$. The observed X-ray flux density at 1 $\mathrm{keV}$ is $(21 \pm 3) \mathrm{nJy}$. The ratio of optical to X-ray flux densities thus evaluates to 29.0 \pm 9.7.

The IC/CMB models developed in the previous section were adapted to return the ratio of optical to X-ray flux as a function of $\gamma_{\min }$. The effect of the uncertainty on the power law index measurement was included by always calculating the minimum ratio over the confidence region of the power law index. The calculation of the ratio in the case of the flat electron distribution below $\gamma_{\text {min }}$ requires the caveat that the assumption $\gamma \gg 1$ is violated, as $\gamma_{0} \sim 3$ for energies corresponding to the optical data point.

In all cases, the ratio is monotonically increasing with decreasing $\gamma_{\min }$ as long as the departure from power law behavior occurs at an energy below the reference X-ray energy, i.e. when $\gamma_{\min } \delta_{10} \lesssim 75$ as determined by the spectral investigation above. For $\gamma_{\min } \delta_{10}=50$, the ratio evaluates to less than 10 and is safely below the observed ratios quoted above. Lower limits on $\gamma_{\min }$ are thus obtained by inverting the optical/X-ray ratio vs. $\gamma_{\text {min }}$ relation and reading off $\gamma_{\min }$ at the appropriate statistical upper limits on the ratio. The lower limits on $\gamma_{\min }$ thus obtained will actually underestimate the true lower limits, since the two-sided errors on the optical to X-ray flux ratio, and not the one-sided upper limits, are used.

Table 3 summarizes the lower limits on $\gamma_{\min }$ thus obtained. Note that at $99 \%$ confidence, the lower limit in all cases relaxes to the minimum possible value for $\gamma_{\min }$ of 1 . 


\section{DISCUSSION}

Tables 2 and 3 summarize the constraints on $\gamma_{\min }$ that we are able to place. If the full bandpass of $0.3-7 \mathrm{keV}$ is used, marginal evidence for a break in the spectrum at around $\gamma_{\min } \delta_{10}=40-55$ emerges, depending on the model for the electron distribution and the kernel for IC scattering. Given the uncertainties with the low-energy calibration of Chandra, the bins below $0.55 \mathrm{keV}$ might want to be excluded from analysis, in which case only upper limits can be placed on $\gamma_{\min }$ from the X-ray spectrum alone: $\gamma_{\min } \delta_{10} \lesssim 80$.

A spurious break due to $\gamma_{\text {min }}$ might be detected if the column of Galactic absorption used in the fit is an under-estimate. However, $n_{\mathrm{H}}$ would need to be increased to about $1.4 \times 10^{21} \mathrm{~cm}^{-2}$ (an increase of over $50 \%$ ) to make the fitting statistic increase monotonically with $\gamma_{\text {min }}$. The behavior of $n_{\mathrm{H}}$ in the vicinity of PKS 0637-752 was investigated by visually inspecting the IRAS $100 \mu \mathrm{m}$ map (Wheelock et al. 1994). No evidence for any Galactic molecular clouds was found that would average out in the large-beam radio surveys resulting in a biased value for the quasar's $n_{\mathrm{H}}$ reported in Dickey \& Lockman (1990). The difference between their value and the one reported in the Leiden/Argentine/Bonn Survey of Galactic HI (Kalberla et al. 2005) is only $15 \%$ (the former being the higher value); it is therefore unlikely that uncertainties in the $n_{\mathrm{H}}$ measurement alone are responsible for the minimum in the fitting statistic.

In Figure 4, it can be seen that, for the same value of $\gamma_{\min }$, the point at which the spectrum deviates from power law shape is at a lower energy for the Aharonian \& Atovan (1981) kernel for inverse Compton scattering compared to the Blumenthal \& Gould (1970) kernel, by about a factor of 3 . This results in tighter lower limits on $\gamma_{\min }$ for the Aharonian \& Atovan (1981) kernel, but correspondingly less-strict upper limits.

The Aharonian \& Atovan (1981) kernel is the more appropriate one for the situation of a jet moving relativistically through a homogeneous and isotropic distribution of seed photons, as is the case for the cosmic microwave background. The comparison to the other kernel can serve to illustrate the order of magnitude of the uncertainty introduced by the choice of the inverse Compton scattering formalism.

We only investigate two possible shapes of the cutoff at $\gamma_{\min }$ : a sharp cutoff such that there are no electrons below $\gamma_{\text {min }}$ (case 1 in Figure 4), and an electron distribution that has a constant density below $\gamma_{\min }$ (case 2 in the same figure). The real shape of the cutoff is likely to be more complicated than in either of these models, but these two distributions bracket the range of expected real distributions, where the cutoff is probably less sharp than in case 1 , but the electron distribution does cut off more quickly than to a constant distribution below $\gamma_{\text {min }}$. As expected, if the cutoff is sharp, the fitting statistic increases rapidly as $\gamma_{\min }$ 
increases, while the increase is slower for the case of the flat distribution below $\gamma_{\min }$, since the X-ray spectrum in this case exhibits a correspondingly milder cutoff.

With $\gamma_{\min } \delta_{10} \lesssim 80$, and assuming $\Gamma=\delta$, the equipartition magnetic field strength for a jet composed of hot electrons and cold protons is $\approx 10 \mu \mathrm{G}$ (Dermer \& Atoyan 2004), and the radio spectrum is expected to extend unbroken down into the hundreds of $\mathrm{kHz}$ (Schwartz 2007).

Georganopoulos et al. (2005), based on the work of Dermer \& Atoyan (2004), calculate the kinetic power in the PKS 0637-752 jet as a function of $\gamma_{\min }$ for the two extreme cases of a hadronic jet (equal numbers of electrons and [cold] protons) and a purely leptonic jet, assuming that $\mathrm{IC} / \mathrm{CMB}$ is the dominant emission mechanism for the $\mathrm{X}$-rays, and that $\Gamma=\delta$. Our limit of $\gamma_{\min } \delta_{10} \lesssim 80$ is consistent with the ranges in $\gamma_{\min }$ considered in their paper, for both the leptonic and hadronic jet, and allows for a jet power of around $5 \times 10^{46} \mathrm{erg} \mathrm{s}^{-1}$ for a hadronic jet and $<10^{46} \mathrm{erg} \mathrm{s}^{-1}$ for a leptonic jet. Estimations of the jet power in earlier work, such as Tavecchio et al. (2000) $\left(L=3 \times 10^{48} \mathrm{erg} \mathrm{s}^{-1}\right.$ for $\left.\gamma_{\text {min }}=10\right)$, Celotti. Ghisellini \& Chiaberge $(2001)\left(L=8 \times 10^{47} \mathrm{erg} \mathrm{s}^{-1}\right.$ for $\left.\gamma_{\text {min }}=10-20\right)$, or Uchivama et al. (2005) $\left(L=9 \times 10^{46} \mathrm{erg} \mathrm{s}^{-1}\right.$ for $\left.\gamma_{\min }=20\right)$ are likely to be too high, given that $\gamma_{\min }$ can in fact be higher than the assumed values without violating the X-ray spectral data. Note also that the limits $10 \lesssim \gamma_{\text {min }} \lesssim 40$ in Uchiyama et al. (2005) are too restrictive in light of our results.

For the adopted jet angle with respect to the line of sight of $6.4^{\circ}$, the deprojected length of the X-ray jet is on the order of $900 \mathrm{kpc}$. With the estimated magnetic field strength of 10 $\mu \mathrm{G}$, the cooling time (taking both IC/CMB and synchrotron losses into account) for electrons with $\gamma \lesssim 8,500$ is larger than the travel time along the jet (Equation D1 in Stawarz et al. 2004). It would be possible therefore to produce these electrons in the central core and propagate them at the bulk velocity to the sites of X-ray emission.

The high contrast between the X-ray knot and the inner jet in PKS 0637-752 argues against the presence of a significant population of electrons with Lorentz factors in the range from $\approx 100-1000$ in the inner jet region at the current epoch. Whether the morphology is due to modulated jet activity or to localized particle acceleration sites remains unresolved.

\section{CONCLUSION}

As far as we are aware, this work represents the first attempt at placing confidence limits on the low-energy electron distribution cutoff in a jet based on a fit to its X-ray spectrum. Usually, papers investigating the broad-band spectra of jet sources simply quote a value for 
$\gamma_{\text {min }}$ that happens to make the model pass through the measured data points. It is very hard to conduct observations in the radio that are able to constrain $\gamma_{\min }$ Gopal-Krishna, Biermann \& Wiita 2004), especially for the kpc-scale jets, where the frequency at which the cutoff due to $\gamma_{\min }$ would manifest itself is below the synchrotron self-absorption frequency. Thus, the soft X-ray spectrum as well as any measured optical fluxes are the best tools to shed light on the behavior of the low-energy end of the electron distribution.

We find that $\gamma_{\min } \delta_{10} \lesssim 80$, which is significantly higher than the value of $10-20$ that has previously been assumed in broad-band spectral modeling of this source. The kinetic power requirement is therefore lessened, but the questions of jet composition and X-ray emission mechanism are not addressed conclusively with this finding.

The present work did not consider other proposed emission mechanisms for the X-ray spectrum, such as direct synchrotron or synchrotron self-Compton. Based on the above considerations, however, IC/CMB remains a viable model for the X-ray emission in PKS 0637-752.

This research was supported in part by NASA Contract NAS8-39073 to the Chandra X-ray Center and CXC grant GO3-4120X to SAO, as well as by the Department of Energy Contract DE-AC02-76SF00515 to the Stanford Linear Accelerator Center. The data reduction made use of the Chandra Interactive Analysis of Observations tools (http://cxc.harvard.edu/ciao), version 3.3, CALDB version 3.2.4. Spectral fits were obtained in XSPEC (Arnaud 1996). We wish to thank the anonymous referee for many helpful comments, and Mark Bautz and Catherine Grant for assistance understanding the low-energy response of Chandra. Further thanks to Greg Madejski and Eukasz Stawarz for fruitful discussions at all stages of this work.

\section{REFERENCES}

Aharonian, F. A. \& Atoyan, A. M. 1981, Ap\&SS, 79, 321

Arnaud, K. A. 1996, in ASP Conf. Ser. 101, Astronomical Data Analysis Software and Systems V, ed. G. Jacoby \& J. Barnes (Provo, UT: Astronomical Society of the Pacific), 17

Blumenthal, G. R. \& Gould, R. J. 1970, RevModPhys, 42, 237

Celotti, A., Ghisellini, G., \& Chiaberge, M. 2001, MNRAS, 321, L1

Chartas, G. et al. 2000, ApJ, 542, 655 
Dermer, C. D. \& Atoyan, A. 2004, ApJ, 611, L9

Dickey, J. M. \& Lockman, F. J. 1990, ARAA, 28, 215

Felten, J. E., Morrison, P. 1966, ApJ, 146, 686

Fixsen, D. J., Cheng, E. S., Gales, J. M., Mather, J. C., Shafer, R. A., \& Wright, E. L. 1996, ApJ, 473, 576

Georganopoulos, M., Kazanas, D., Perlman, E., \& Stecker, F. W. 2005, ApJ, 625, 656

Gopal-Krishna, Biermann, P. L., \& Wiita, P. J. 2004, ApJ, 603, L9

Harris, D. E. \& Krawczynski, H. 2002, ApJ, 565, 244

Kalberla, P. M. W., Burton, W. B., Hartmann, Dap, Arnal, E. M., Bajaja, E., Morras, R., \& Pppel, W. G. L. 2005, A\&A, 440, 775

Lovell, J. E. J. et al. 2000, in Astrophysical Phenomena Revealed by Space VLBI, ed. H. Hirabayashi, P. G. Edwards, \& D. W. Murphy (Sagamihara, Japan: ISAS), 215

Marshall, H. L. et al. 2005, ApJS, 156, 13

Press, W. H., Teukolsky, S. A., Vetterling, W. T., Flannery, B. P. 1992, Numerical Recipes in C (Cambridge, U.K.: Cambridge Univ. Press)

Rybicki, G. B. \& Lightman, A. P. 1979, Radiative Processes in Astrophysics (New York, NY: John Wiley \& Sons)

Sambruna, R. M. et al. 2002, ApJ, 571, 206

Sambruna, R. M. et al. 2004, ApJ, 608, 698

Sambruna, R. M. et al. 2006, ApJ, 641, 717

Savage, A., Browne, I.W.A., \& Bolton, J. G. 1976, MNRAS, 177, 77P

Schwartz, D. A. et al. 2000, ApJ 540, L69

Schwartz, D. A. et al. 2006, ApJ 650, 592

Schwartz, D. A. 2007, in Revista Mexicana de Astronomía y Astrofísica 27, Triggering Relativistic Jet, ed. W. H. Lee \& E. Ramírez-Ruiz (Mexico City, Mexico: UNAM), 102

Siemiginowska, A., Bechtold, J., Aldcroft, T. L., Elvis, M., Harris, D. E., \& Dobrzycki, A. 2002, ApJ, 570, 543 
Siemiginowska, A., Smith, R. K., Aldcroft, T. L., Schwartz, D. A., Paerels, F., \& Petric, A. O. 2003, ApJ, 598, L15

Stawarz, Ł., Sikora, M., Ostrowski, M., Begelman, M. C. 2004, ApJ, 608, 95

Stawarz, Ł., Siemiginowska, A., Ostrowski, M., \& Sikora, M. 2005, ApJ, 626, 120

Tavecchio, F., Maraschi, L., Sambruna, R. M., Urry, C. M. 2000, ApJ, 544, L23

Tingay, S. J. et al. 1998, ApJ, 497, 594

Uchiyama, Y. et al. 2005, ApJ, 631, L113

Wheelock, S.-L. et al. 1994, IRAS Sky Survey Atlas, JPL Publication 94-11 (Pasadena: Jet Propulsion Laboratory) 
Table 1. Chandra ACIS-S Observations of PKS 0637-752 Used in this Investigation ${ }^{\mathrm{a}}$

\begin{tabular}{|c|c|c|c|}
\hline ObsID & $\begin{array}{l}\text { Exposure } \\
\text { Time (ks) }\end{array}$ & ObsID & $\begin{array}{l}\text { Exposure } \\
\text { Time (ks) }\end{array}$ \\
\hline 472 & 5.52 & 1062 & 0.62 \\
\hline 473 & 3.54 & 1063 & 1.22 \\
\hline 474 & 4.64 & 62549 & 6.25 \\
\hline 475 & 4.64 & 62550 & 5.20 \\
\hline 476 & 2.39 & 62551 & 5.32 \\
\hline 1051 & 1.02 & 62552 & 5.07 \\
\hline 1052 & 1.02 & 62553 & 4.88 \\
\hline 1055 & 2.01 & 62554 & 11.22 \\
\hline 1056 & 1.73 & 62555 & 4.91 \\
\hline 1058 & 1.51 & 62556 & 4.84 \\
\hline 1060 & 0.62 & Total & 78.20 \\
\hline
\end{tabular}

aMore information on the individual observations is available in Table 1 of Chartas et al. (2000). 
Table 2. Constraints on $\gamma_{\text {min }}$ Obtained from the X-ray Spectrum

\begin{tabular}{|c|c|c|c|c|c|}
\hline $\begin{array}{c}\text { X-ray } \\
\text { bandpass }\end{array}$ & $\begin{array}{c}\mathrm{IC} / \mathrm{CMB} \text { kernel } \\
\text { or power law model }\end{array}$ & $\begin{array}{l}\mathrm{e}^{-} \\
\text {distr. }^{\mathrm{b}}\end{array}$ & $\begin{array}{c}\text { best-fit } \\
\text { C-statistic }\end{array}$ & $\begin{array}{c}\gamma_{\min } \delta_{10} \\
(1 \sigma)\end{array}$ & $\begin{array}{c}\gamma_{\min } \delta_{10} \\
(99 \%)\end{array}$ \\
\hline \multirow[t]{5}{*}{$0.3-7 \mathrm{keV}$} & power law & $\ldots$ & 886.3 & & \\
\hline & BluGou & case $(1)$ & 879.4 & $49 \pm 2$ & $49 \pm 6$ \\
\hline & & case (2) & 881.5 & $57 \pm 4$ & $<67$ \\
\hline & AhaAto & case $(1)$ & 881.8 & $63 \pm 2$ & $<67$ \\
\hline & & case $(2)$ & 880.2 & $72 \pm 3$ & $72 \pm 10$ \\
\hline \multirow[t]{5}{*}{$0.55-7 \mathrm{keV}$} & power law & $\ldots$ & 817.2 & & \\
\hline & BluGou & case $(1)$ & 818.6 & $<50$ & $<54$ \\
\hline & & case $(2)$ & 817.2 & $<55$ & $<63$ \\
\hline & AhaAto & case (1) & 817.3 & $<67$ & $<70$ \\
\hline & & case $(2)$ & 817.3 & $<73$ & $<79$ \\
\hline
\end{tabular}

${ }^{\mathrm{a} B l u G o u}=$ Blumenthal \& Gould $(1970)$, AhaAto $=$ Aharonian \& Atovan (1981)

${ }^{\mathrm{b}}$ Cases (1) and (2) refer to the different electron distributions in Figure 4.

Table 3. Lower Limits on $\gamma_{\text {min }}$ Obtained from Optical and X-ray Flux Measurement

\begin{tabular}{|c|c|c|c|}
\hline IC/CMB kernel ${ }^{\mathrm{a}}$ & $\begin{array}{c}\mathrm{e}^{-} \\
\text {distr. }^{\mathrm{b}}\end{array}$ & $\begin{array}{c}\gamma_{\min } \delta_{10} \\
(1 \sigma)\end{array}$ & $\begin{array}{c}\gamma_{\min } \delta_{10} \\
(99 \%)\end{array}$ \\
\hline \multirow[t]{2}{*}{ BluGou } & case (1) & $>4.2$ & $>1$ \\
\hline & case $(2)$ & $>6.1$ & $>1$ \\
\hline \multirow[t]{2}{*}{ AhaAto } & case (1) & $>4.8$ & $>1$ \\
\hline & case $(2)$ & $>7.7$ & $>1$ \\
\hline${ }^{\mathrm{a} B l u G o u}=$ & \multicolumn{2}{|c|}{ Blumenthal \& Gould } & $(1970)$ \\
\hline \multicolumn{4}{|c|}{ AhaAto $=$ Aharonian \& Atoyan $(1981)$} \\
\hline
\end{tabular}




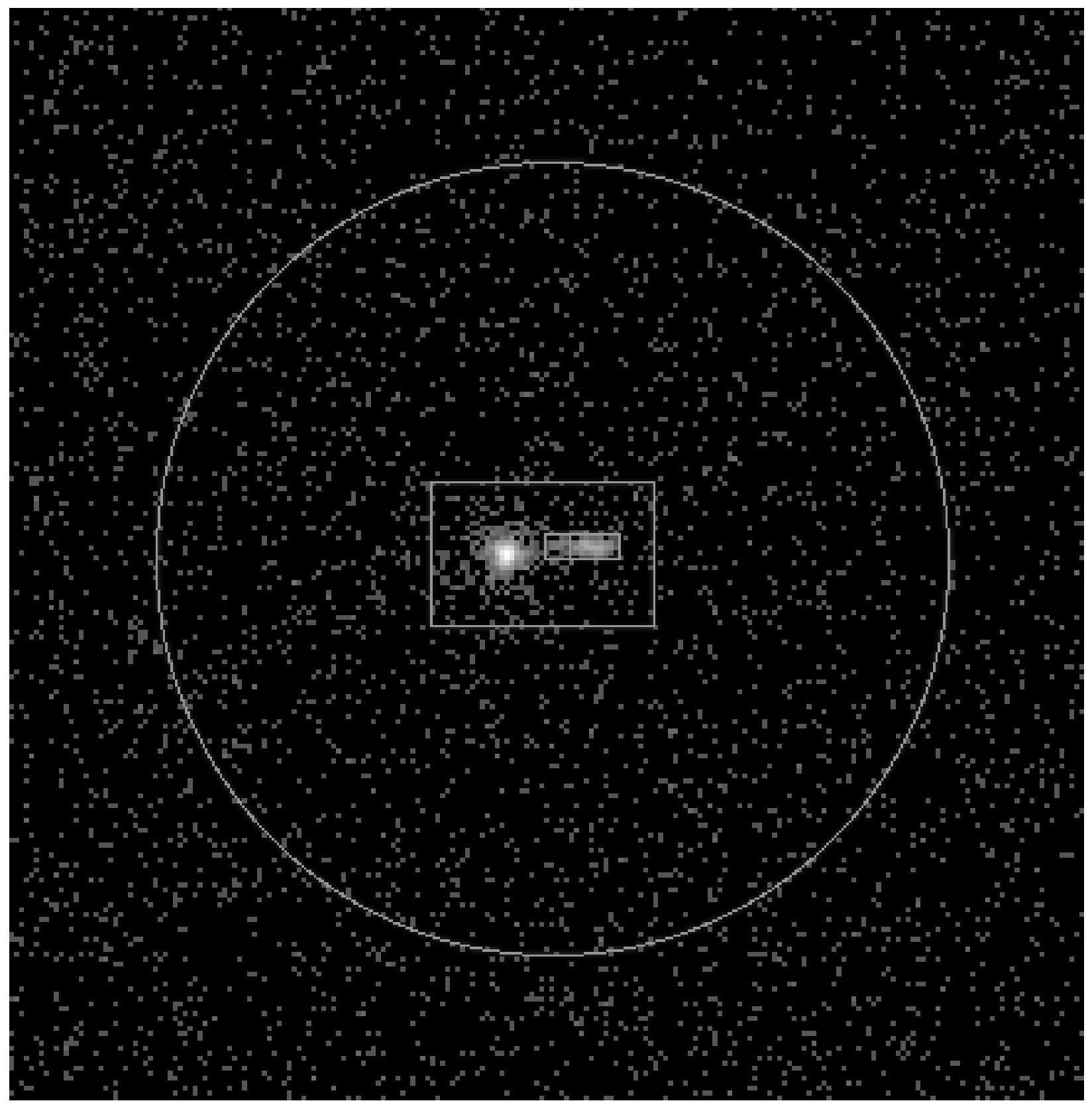

Fig. 1. - Extraction regions superimposed on one of the exposures of PKS 0637-752 (ObsID 62554). The inner and outer jet regions are the two rectangular regions straddling the jet; the background was extracted from the elliptical region while excluding the counts from within the larger rectangular box. These extraction regions are representative of the regions used for all observations; however, the change in focus for some of them necessitated larger boxes for the inner and outer jet, which were then chosen to again just straddle the image of the jet. 


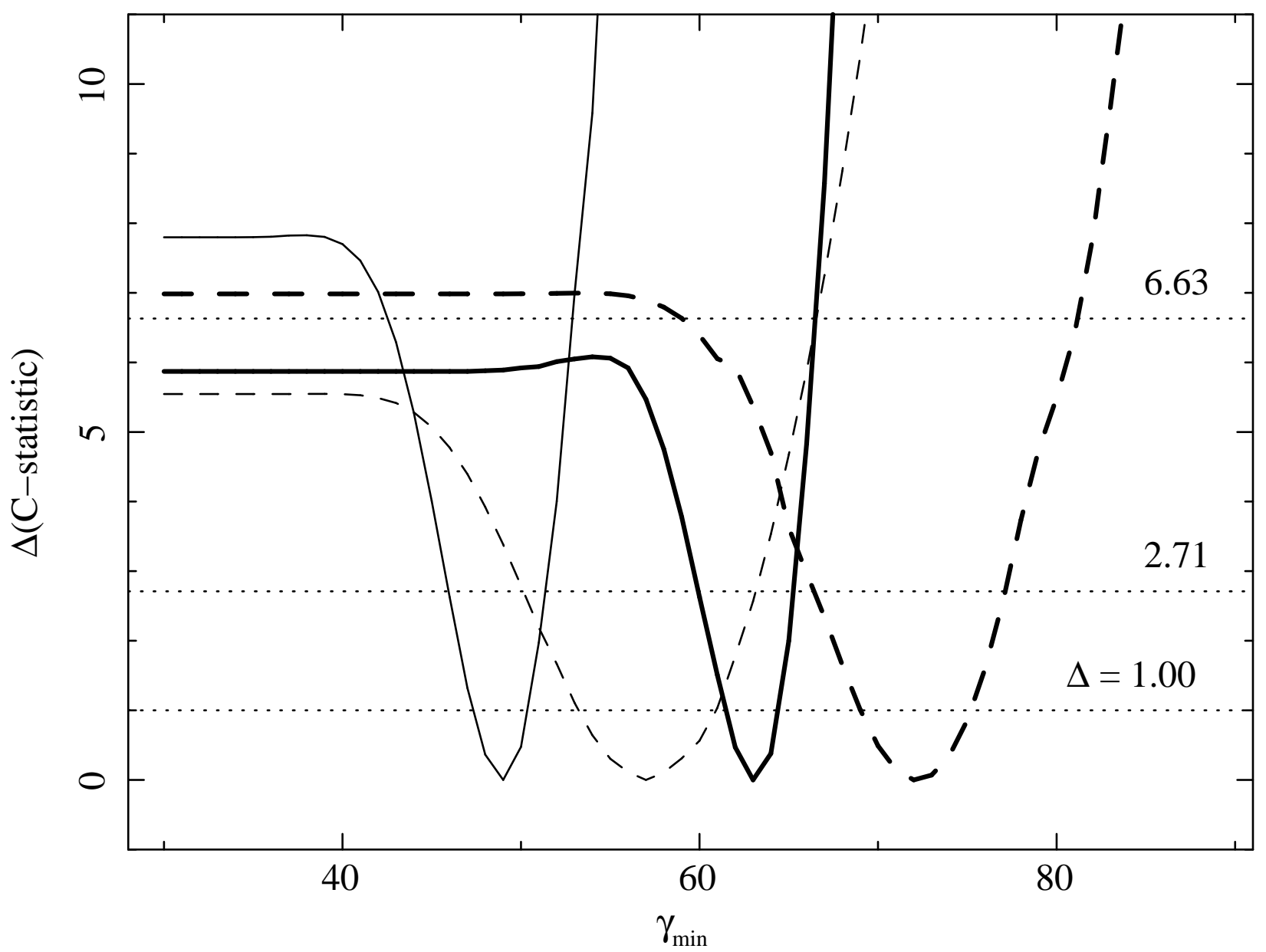

Fig. 2.- Confidence limits on $\gamma_{\min }$ for the IC/CMB model fits, obtained from the $0.3-7 \mathrm{keV}$ X-ray spectrum. The bold lines are for the Aharonian \& Atoyan (1981) kernel, the thin lines for the Blumenthal \& Gould (1970) kernel. In both cases, the solid line is for the electron distribution with the sharp cutoff at $\gamma_{\min }$ (case (1) in Figure 4), and the dashed line for the constant segment below $\gamma_{\min }$ (case (2) in Figure 4 ). The three dotted lines mark the $\Delta($ C-statistic $)=1.00,2.71$ and 6.63 levels corresponding to the $68.3,90$ and $99 \%$ two-sided confidence limits on $\gamma_{\min }$. 


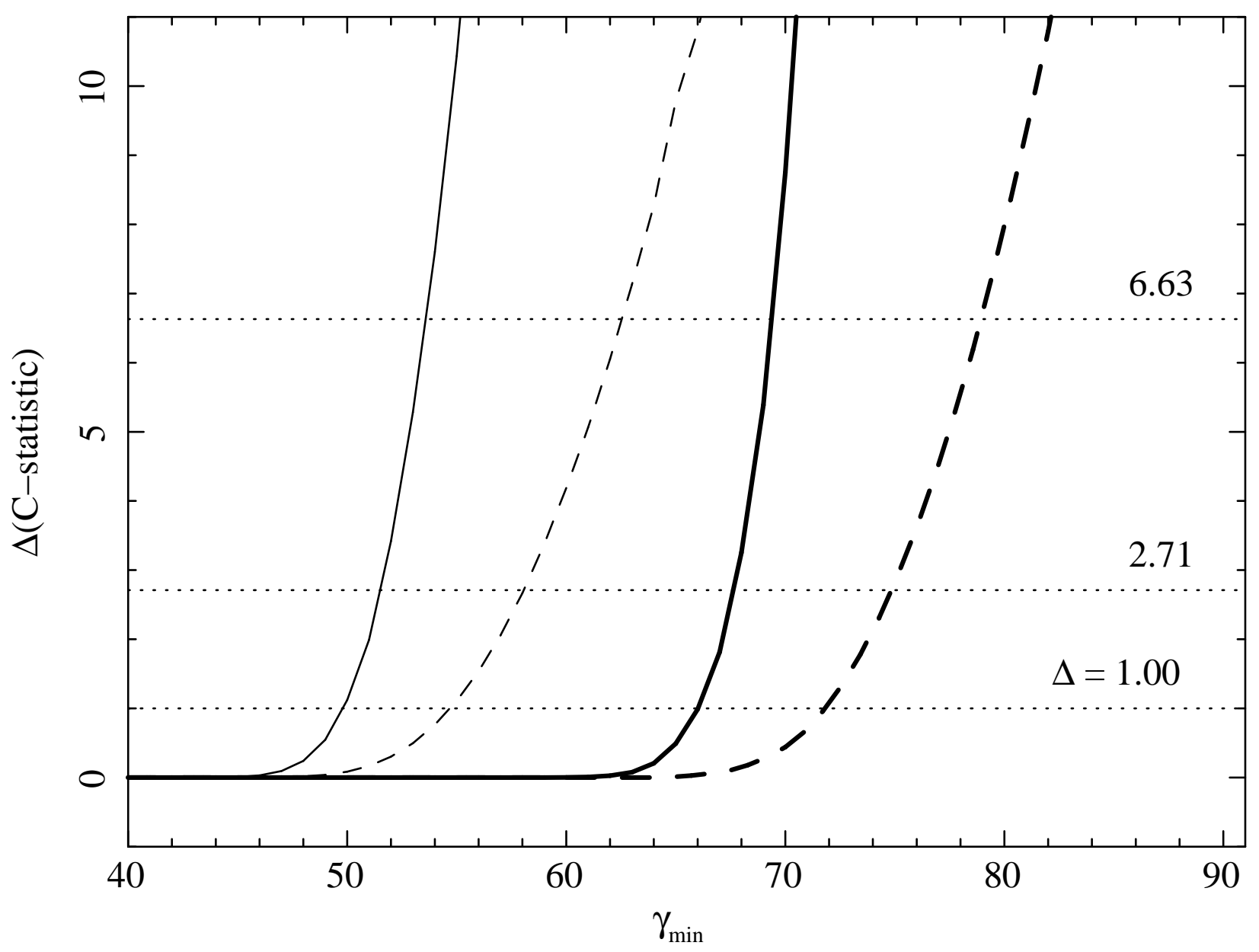

Fig. 3.- Confidence limits on $\gamma_{\min }$ for the IC/CMB model fits, obtained from the X-ray spectrum using the restricted $0.55-7 \mathrm{keV}$ bandpass. The same conventions as for Figure 2 apply. 


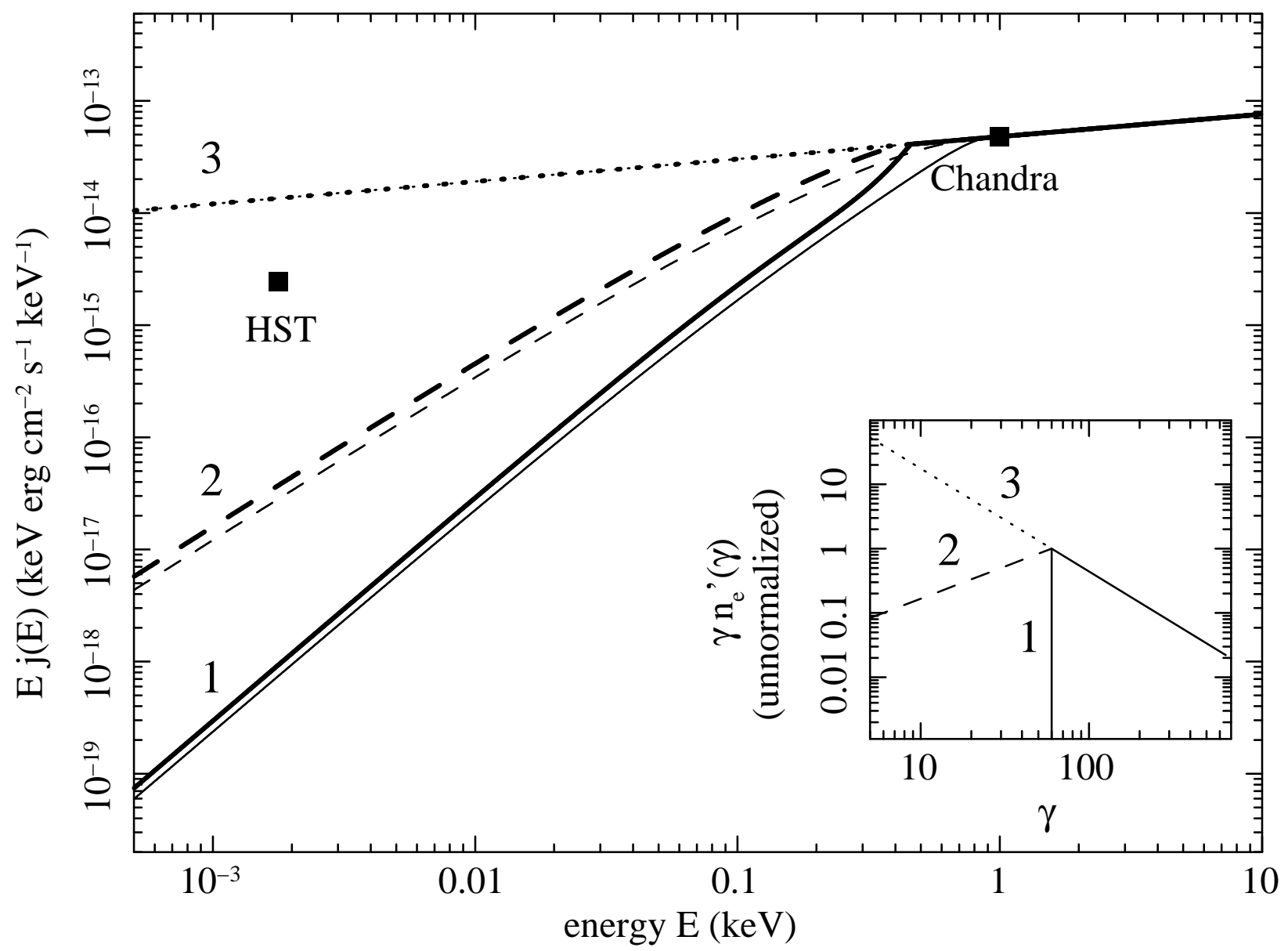

Fig. 4. - Predicted flux of the IC/CMB models developed in Section 3, The adjustable parameters are set to $\gamma_{\min }=60$ and $s=2.6$. The inset plot shows the selected electron density distributions, which are all described as a power law with slope $s$ above $\gamma_{\min }$. Note that the normalization of the $\mathrm{y}$ axis is arbitrary. The two cases with a modification of the spectrum at $\gamma_{\min }$ are characterized by either the step-function cutoff at $\gamma_{\min }(1)$ or the constant density below $\gamma_{\min }(2)$. Case 3 results when $\gamma_{\min }$ is chosen sufficiently low such that the cutoff in the photon spectrum moves outside its bandpass. The spectra based on the Aharonian \& Atovan (1981) kernel are shown as bold lines, the ones based on the Blumenthal \& Gould (1970) kernel as thin lines. The spectra for the two models coincide for case (3). Both sets of spectra have been normalized to the observed X-ray flux. The optical data point is also plotted and is violated by the models without a cutoff in the electron distribution. The allowable range of $\gamma_{\min }$, such that the cutoff occurs below the $\mathrm{X}$-ray data point, and the optical flux from the IC/CMB model does not over-predict the optical detection, is approximately $5-80$. 